CERN-PPE/94-98

28 June 1994

\title{
Search for Rare Hadronic B Decays
}

\author{
The OPAL Collaboration
}

\begin{abstract}
A search for rare decays of $B$ hadrons has been performed using approximately two million hadronic $\mathrm{Z}^{0}$ decays collected by the OPAL experiment between 1990 and 1993 . The exclusive decay channels $\mathrm{B}_{\mathrm{d}}^{0} \rightarrow \pi^{+} \pi^{-}, \mathrm{B}_{\mathrm{d}}^{0} \rightarrow \mathrm{K}^{+} \pi^{-}, \mathrm{B}_{\mathrm{s}}^{0} \rightarrow \pi^{+} \mathrm{K}^{-}$and $\mathrm{B}_{\mathrm{s}}^{0} \rightarrow \mathrm{K}^{+} \mathrm{K}^{-}$have been studied. Such decays include contributions from flavour changing neutral current processes such as hadronic penguin decays. No significant excess of events has been observed in the $\pi^{+} \pi^{-}, \pi^{+} \mathrm{K}^{-}$and $\mathrm{K}^{+} \mathrm{K}^{-}$invariant mass distributions. Consequently, upper limits on the $\mathrm{B}_{\mathrm{d}}^{0}$ branching ratios,

$$
\operatorname{Br}\left(\mathrm{B}_{\mathrm{d}}^{0} \rightarrow \pi^{+} \pi^{-}\right)<4.7 \times 10^{-5} \text { and } \operatorname{Br}\left(\mathrm{B}_{\mathrm{d}}^{0} \rightarrow \mathrm{K}^{+} \pi^{-}\right)<8.1 \times 10^{-5},
$$

and on the $\mathrm{B}_{\mathrm{s}}^{0}$ branching ratios,

$$
\operatorname{Br}\left(\mathrm{B}_{\mathrm{s}}^{0} \rightarrow \pi^{+} \mathrm{K}^{-}\right)<2.6 \times 10^{-4} \text { and } \operatorname{Br}\left(\mathrm{B}_{\mathrm{s}}^{0} \rightarrow \mathrm{K}^{+} \mathrm{K}^{-}\right)<1.4 \times 10^{-4},
$$

have been set at the $90 \%$ confidence level. These limits assume that the ratio of the partial decay widths of the $\mathrm{Z}^{0}, \Gamma\left(\mathrm{Z}^{0} \rightarrow \mathrm{b} \overline{\mathrm{b}}\right) / \Gamma\left(\mathrm{Z}^{0} \rightarrow\right.$ hadrons $)$, is 0.217 and that the fractions of $\mathrm{B}_{\mathrm{d}}^{0}$ and $\mathrm{B}_{\mathrm{s}}^{0}$ mesons produced during fragmentation are $39.5 \%$ and $12 \%$ respectively. The $\mathrm{B}_{\mathrm{s}}^{0}$ branching ratio limits are the first such limits obtained.
\end{abstract}




\section{The OPAL Collaboration}

R. Akers ${ }^{16}$, G. Alexander ${ }^{23}$, J. Allison ${ }^{16}$, K.J. Anderson ${ }^{9}$, S. Arcelli ${ }^{2}$, S. Asai ${ }^{24}$, A. Astbury ${ }^{28}$, D. Axen ${ }^{29}$, G. Azuelos ${ }^{18, a}$, A.H. Ball ${ }^{17}$, E. Barberio ${ }^{26}$, R.J. Barlow ${ }^{16}$, R. Bartoldus ${ }^{3}$, J.R. Batley ${ }^{5}$, G. Beaudoin ${ }^{18}$, A. Beck ${ }^{23}$, G.A. Beck ${ }^{13}$, J. Becker ${ }^{10}$, C. Beeston ${ }^{16}$, T. Behnke ${ }^{27}$, K.W. Bell ${ }^{20}$, G. Bella ${ }^{23}$, P. Bentkowski ${ }^{18}$, S. Bentvelsen ${ }^{8}$, P. Berlich ${ }^{10}$, S. Bethke ${ }^{32}$, O. Biebel ${ }^{32}$, I.J. Bloodworth ${ }^{1}$, P. Bock ${ }^{11}$, H.M. Bosch ${ }^{11}$, M. Boutemeur ${ }^{18}$, S. Braibant ${ }^{12}$, P. Bright-Thomas ${ }^{25}$, R.M. Brown ${ }^{20}$, A. Buijs ${ }^{8}$, H.J. Burckhart ${ }^{8}$, C. Burgard ${ }^{27}$, P. Capiluppi ${ }^{2}$, R.K. Carnegie ${ }^{6}$, A.A. Carter $^{13}$, J.R. Carter ${ }^{5}$, C.Y. Chang ${ }^{17}$, C. Charlesworth ${ }^{6}$, D.G. Charlton ${ }^{8}$, S.L. Chu ${ }^{4}$, P.E.L. Clarke ${ }^{15}$, J.C. Clayton ${ }^{1}$, S.G. Clowes ${ }^{16}$, I. Cohen ${ }^{23}$, J.E. Conboy ${ }^{15}$, M. Coupland ${ }^{14}$, M. Cuffiani ${ }^{2}$, S. Dado ${ }^{22}$, C. Dallapiccola ${ }^{17}$, G.M. Dallavalle ${ }^{2}$, C. Darling ${ }^{31}$, S. De Jong ${ }^{13}$, H. Deng ${ }^{17}$, M. Dittmar ${ }^{4}$, M.S. Dixit ${ }^{7}$, E. do Couto e Silva ${ }^{12}$, J.E. Duboscq ${ }^{8}$, E. Duchovni ${ }^{26}$, G. Duckeck ${ }^{8}$, I.P. Duerdoth ${ }^{16}$, U.C. Dunwoody ${ }^{5}$, P.A. Elcombe ${ }^{5}$, P.G. Estabrooks ${ }^{6}$, E. Etzion ${ }^{23}$, H.G. Evans ${ }^{9}$, F. Fabbri ${ }^{2}$, B. Fabbro ${ }^{21}$, M. Fanti ${ }^{2}$, M. Fierro ${ }^{2}$, M. Fincke-Keeler ${ }^{28}$, H.M. Fischer ${ }^{3}$, P. Fischer ${ }^{3}$, R. Folman ${ }^{26}$, D.G. Fong ${ }^{17}$, M. Foucher ${ }^{17}$, H. Fukui ${ }^{24}$, A. Fürtjes ${ }^{8}$, P. Gagnon ${ }^{6}$,

A. Gaidot ${ }^{21}$, J.W. Gary ${ }^{4}$, J. Gascon ${ }^{18}$, N.I. Geddes ${ }^{20}$, C. Geich-Gimbel ${ }^{3}$, S.W. Gensler ${ }^{9}$, F.X. Gentit ${ }^{21}$, T. Geralis ${ }^{20}$, G. Giacomelli ${ }^{2}$, P. Giacomelli ${ }^{4}$, R. Giacomelli ${ }^{2}$, V. Gibson ${ }^{5}$, W.R. Gibson ${ }^{13}$, J.D. Gillies ${ }^{20}$, J. Goldberg ${ }^{22}$, D.M. Gingrich ${ }^{30, a}$, M.J. Goodrick ${ }^{5}$, W. Gorn ${ }^{4}$, C. Grandi ${ }^{2}$, P. Grannis ${ }^{8}$, E. Gross ${ }^{26}$, J. Hagemann ${ }^{27}$, G.G. Hanson ${ }^{12}$, M. Hansroul ${ }^{8}$, C.K. Hargrove ${ }^{7}$, J. Hart ${ }^{8}$, P.A. Hart ${ }^{9}$, M. Hauschild ${ }^{8}$, C.M.Hawkes ${ }^{8}$, E. Heflin ${ }^{4}$,

R..J.Hemingway ${ }^{6}$, G. Herten ${ }^{10}$, R.D. Heuer ${ }^{8}$, J.C.Hill ${ }^{5}$, S.J. Hillier ${ }^{8}$, T. Hilse ${ }^{10}$, D.A. Hinshaw ${ }^{18}$, P.R. Hobson ${ }^{25}$, D. Hochman ${ }^{26}$, A. Höcker ${ }^{3}$, R.J. Homer ${ }^{1}$, A.K. Honma ${ }^{28, a}$, R.E. Hughes-Jones ${ }^{16}$, R. Humbert ${ }^{10}$, P. Igo-Kemenes ${ }^{11}$, H. Ihssen ${ }^{11}$, D.C. Imrie ${ }^{25}$, A. Jawahery ${ }^{17}$, P.W. Jeffreys ${ }^{20}$,

H. Jeremie ${ }^{18}$, M. Jimack ${ }^{1}$, M. Jones ${ }^{6}$, R.W.L. Jones ${ }^{8}$, P. Jovanovic ${ }^{1}$, C. Jui ${ }^{4}$, D. Karlen ${ }^{6}$,

K. Kawagoe $^{24}$, T. Kawamoto ${ }^{24}$, R.K. Keeler ${ }^{28}$, R.G. Kellogg ${ }^{17}$, B.W. Kennedy ${ }^{20}$, B. King ${ }^{8}$, J. King ${ }^{13}$, S. Kluth ${ }^{5}$, T. Kobayashi ${ }^{24}$, M. Kobel ${ }^{10}$, D.S. Koetke ${ }^{8}$, T.P. Kokott ${ }^{3}$, S. Komamiya ${ }^{24}$, R. Kowalewski ${ }^{8}$, R. Howard ${ }^{29}$, P. Krieger ${ }^{6}$, J. von Krogh ${ }^{11}$, P. Kyberd ${ }^{13}$, G.D. Lafferty ${ }^{16}$,

H. Lafoux ${ }^{8}$, R. Lahmann ${ }^{17}$, J. Lauber ${ }^{8}$, J.G. Layter ${ }^{4}$, P. Leblanc ${ }^{18}$, P. Le Du ${ }^{21}$, A.M. Lee ${ }^{31}$, E. Lefebvre ${ }^{18}$, M.H. Lehto ${ }^{15}$, D. Lellouch ${ }^{26}$, C. Leroy ${ }^{18}$, J. Letts ${ }^{4}$, L. Levinson ${ }^{26}$, Z. Li ${ }^{12}$, F. Liu ${ }^{29}$, S.L. Lloyd ${ }^{13}$, F.K. Loebinger ${ }^{16}$, G.D. Long ${ }^{17}$, B. Lorazo $^{18}$, M.J. Losty ${ }^{7}$, X.C. Lou ${ }^{8}$, J. Ludwig ${ }^{10}$, A. Luig $^{10}$, M. Mannelli ${ }^{8}$, S. Marcellini ${ }^{2}$, C. Markus ${ }^{3}$, A.J. Martin ${ }^{13}$, J.P. Martin ${ }^{18}$, T. Mashimo ${ }^{24}$, P. Mättig ${ }^{3}$, U. Maur ${ }^{3}$, J. McKenna ${ }^{29}$, T.J. McMahon ${ }^{1}$, A.I. McNab ${ }^{13}$, J.R. McNutt ${ }^{25}$, F. Meijers ${ }^{8}$, F.S. Merritt ${ }^{9}$, H. $\mathrm{Mes}^{7}$, A. Michelini ${ }^{8}$, R.P. Middleton ${ }^{20}$, G. Mikenberg ${ }^{26}$, J. Mildenberger ${ }^{6}$, D.J. Miller ${ }^{15}$, R. Mir $^{26}$, W. Mohr ${ }^{10}$, C. Moisan ${ }^{18}$,

A. Montanari ${ }^{2}$, T. Mori ${ }^{24}$, M. Morii ${ }^{24}$, U. Müller ${ }^{3}$, B. Nellen ${ }^{3}$, B. Nijjhar ${ }^{16}$, S.W. O’Neale ${ }^{1}$, F.G. Oakham ${ }^{7}$, F. Odorici ${ }^{2}$, H.O. Ogren ${ }^{12}$, C.J. Oram ${ }^{28, a}$, M.J. Oreglia ${ }^{9}$, S. Orito ${ }^{24}$, J.P. Pansart ${ }^{21}$, G.N.Patrick ${ }^{20}$, M.J. Pearce ${ }^{1}$, P. Pfister ${ }^{10}$, P.D. Phillips ${ }^{16}$, J.E. Pilcher ${ }^{9}$,

J. Pinfold ${ }^{30}$, D. Pitman ${ }^{28}$, D.E. Plane ${ }^{8}$, P. Poffenberger ${ }^{28}$, B. Poli ${ }^{2}$, A. Posthaus ${ }^{3}$,

T.W. Pritchard ${ }^{13}$, H. Przysiezniak ${ }^{18}$, M.W. Redmond ${ }^{8}$, D.L. Rees ${ }^{8}$, D. Rigby ${ }^{1}$, M. Rison ${ }^{5}$, S.A. Robins ${ }^{13}$, D. Robinson ${ }^{5}$, J.M. Roney ${ }^{28}$, E. Ros ${ }^{8}$, S. Rossberg ${ }^{10}$, A.M. Rossi ${ }^{2}$, M. Rosvick ${ }^{28}$, P. Routenburg ${ }^{30}$, Y. Rozen ${ }^{8}$, K. Runge ${ }^{10}$, O. Runolfsson ${ }^{8}$, D.R. Rust ${ }^{12}$, M.Sasaki ${ }^{24}$, C.Sbarra ${ }^{2}$, A.D.Schaile ${ }^{8}$, O.Schaile ${ }^{10}$, F. Scharf ${ }^{3}$, P.Scharff-Hansen ${ }^{8}$, P.Schenk ${ }^{4}$, B. Schmitt ${ }^{3}$, H. von der Schmitt ${ }^{11}$, M.Schröder ${ }^{12}$, H.C.Schultz-Coulon ${ }^{10}$, P.Schütz ${ }^{3}$, M.Schulz ${ }^{8}$, C. Schwick ${ }^{27}$, J.Schwiening ${ }^{3}$, W.G. Scott ${ }^{20}$, M.Settles ${ }^{12}$, T.G.Shears ${ }^{5}$, B.C.Shen ${ }^{4}$,

C.H.Shepherd-Themistocleous ${ }^{7}$, P.Sherwood ${ }^{15}$, G.P.Siroli ${ }^{2}$, A.Skillman ${ }^{16}$, A.Skuja ${ }^{17}$, 
A.M.Smith ${ }^{8}$, T.J.Smith ${ }^{28}$, G.A.Snow ${ }^{17}$, R. Sobie $^{28}$, R.W.Springer ${ }^{17}$, M.Sproston ${ }^{20}$, A.Stahl ${ }^{3}$, C.Stegmann ${ }^{10}$, K.Stephens ${ }^{16}$, J.Steuerer ${ }^{28}$, B.Stockhausen ${ }^{3}$, R.Ströhmer ${ }^{11}$, D. Strom ${ }^{19}$, P. Szymanski ${ }^{20}$, H. Takeda ${ }^{24}$, T. Takeshita ${ }^{24}$, S. Tarem ${ }^{26}$, M. Tecchio ${ }^{9}$, P. Teixeira-Dias ${ }^{11}$, N. Tesch ${ }^{3}$, M.A. Thomson ${ }^{15}$, S. Towers ${ }^{6}$, T. Tsukamoto ${ }^{24}$, M.F. Turner-Watson ${ }^{8}$, D. Van den plas $^{18}$, R. Van Kooten ${ }^{12}$, G. Vasseur ${ }^{21}$, M. Vincter $^{28}$, A. Wagner ${ }^{27}$, D.L. Wagner ${ }^{9}$, C.P. Ward ${ }^{5}$, D.R. Ward ${ }^{5}$, J.J. Ward ${ }^{15}$, P.M. Watkins ${ }^{1}$, A.T. Watson ${ }^{1}$, N.K. Watson ${ }^{7}$, P. Weber ${ }^{6}$, P.S. Wells ${ }^{8}$, N. Wermes ${ }^{3}$, B. Wilkens ${ }^{10}$, G.W. Wilson ${ }^{4}$, J.A. Wilson ${ }^{1}$, V-H. Winterer ${ }^{10}$, T. Wlodek ${ }^{26}$, G. Wolf ${ }^{26}$, S. Wotton ${ }^{11}$, T.R. Wyatt ${ }^{16}$, A. Yeaman ${ }^{13}$, G. Yekutieli ${ }^{26}$, M. Yurko ${ }^{18}$, W. Zeuner ${ }^{8}$, G.T. Zorn ${ }^{17}$.

${ }^{1}$ School of Physics and Space Research, University of Birmingham, Birmingham B15 2TT, UK ${ }^{2}$ Dipartimento di Fisica dell' Università di Bologna and INFN, I-40126 Bologna, Italy ${ }^{3}$ Physikalisches Institut, Universität Bonn, D-53115 Bonn, Germany

${ }^{4}$ Department of Physics, University of California, Riverside CA 92521, USA

${ }^{5}$ Cavendish Laboratory, Cambridge CB3 0HE, UK

${ }^{6}$ Carleton University, Department of Physics, Colonel By Drive, Ottawa, Ontario K1S 5B6, Canada

${ }^{7}$ Centre for Research in Particle Physics, Carleton University, Ottawa, Ontario K1S 5B6, Canada

${ }^{8}$ CERN, European Organisation for Particle Physics, CH-1211 Geneva 23, Switzerland

${ }^{9}$ Enrico Fermi Institute and Department of Physics, University of Chicago, Chicago IL 60637, USA

${ }^{10}$ Fakultät für Physik, Albert Ludwigs Universität, D-79104 Freiburg, Germany

${ }^{11}$ Physikalisches Institut, Universität Heidelberg, D-69120 Heidelberg, Germany

${ }^{12}$ Indiana University, Department of Physics, Swain Hall West 117, Bloomington IN 47405 , USA

${ }^{13}$ Queen Mary and Westfield College, University of London, London E1 4NS, UK

${ }^{14}$ Birkbeck College, London WC1E 7HV, UK

${ }^{15}$ University College London, London WC1E 6BT, UK

${ }^{16}$ Department of Physics, Schuster Laboratory, The University, Manchester M13 9PL, UK

${ }^{17}$ Department of Physics, University of Maryland, College Park, MD 20742, USA

${ }^{18}$ Laboratoire de Physique Nucléaire, Université de Montréal, Montréal, Quebec H3C 3J7, Canada

${ }^{19}$ University of Oregon, Department of Physics, Eugene OR 97403, USA

${ }^{20}$ Rutherford Appleton Laboratory, Chilton, Didcot, Oxfordshire OX11 0QX, UK

${ }^{21}$ CEA, DAPNIA/SPP, CE-Saclay, F-91191 Gif-sur-Yvette, France

${ }^{22}$ Department of Physics, Technion-Israel Institute of Technology, Haifa 32000, Israel

${ }^{23}$ Department of Physics and Astronomy, Tel Aviv University, Tel Aviv 69978, Israel

${ }^{24}$ International Centre for Elementary Particle Physics and Department of Physics, University of Tokyo, Tokyo 113, and Kobe University, Kobe 657, Japan

${ }^{25}$ Brunel University, Uxbridge, Middlesex UB8 3PH, UK

${ }^{26}$ Particle Physics Department, Weizmann Institute of Science, Rehovot 76100, Israel

${ }^{27}$ Universität Hamburg/DESY, II Institut für Experimental Physik, Notkestrasse 85, D-22607 Hamburg, Germany

${ }^{28}$ University of Victoria, Department of Physics, P O Box 3055, Victoria BC V8W 3P6, Canada 
${ }^{29}$ University of British Columbia, Department of Physics, Vancouver BC V6T 1Z1, Canada ${ }^{30}$ University of Alberta, Department of Physics, Edmonton AB T6G 2J1, Canada

${ }^{31}$ Duke University, Dept of Physics, Durham, NC 27708-0305, USA

${ }^{32}$ Technische Hochschule Aachen, III Physikalisches Institut, Sommerfeldstrasse 26-28, D-52056 Aachen, Germany

${ }^{a}$ Also at TRIUMF, Vancouver, Canada V6T $2 \mathrm{~A} 3$ 


\section{Introduction}

Searches for rare decays of $\mathrm{B}$ hadrons at LEP, with branching ratios of $\mathcal{O}\left(10^{-4}-10^{-5}\right)$, are now becoming feasible with the large statistics available. Such rare processes include decays of B hadrons into final states which do not contain a charmed quark. In the Standard Model [1], these decays can occur through Cabibbo suppressed $b \rightarrow u$ transitions [2] or through one loop diagrams, such as penguin diagrams, which involve a virtual $W^{ \pm}$boson and a heavy quark [3]. In addition, penguin decays offer a unique window to probe new physics beyond the Standard Model. For example, theories with two Higgs doublets contain new diagrams with a charged Higgs boson that add constructively to the $W^{ \pm}$boson loop diagram [3, 4]. Also, in the minimal supersymmetric extension of the Standard Model there could be contributions from superpartners that affect the decay rates $[3,5]$.

This paper describes the search for rare hadronic decays of the $\mathrm{B}_{\mathrm{d}}^{0}$ and $\mathrm{B}_{\mathrm{s}}^{0}$ mesons in the exclusive decay channels ${ }^{1} \mathrm{~B}_{\mathrm{d}}^{0} \rightarrow \pi^{+} \pi^{-}, \mathrm{B}_{\mathrm{d}}^{0} \rightarrow \mathrm{K}^{+} \pi^{-}, \mathrm{B}_{\mathrm{s}}^{0} \rightarrow \pi^{+} \mathrm{K}^{-}$and $\mathrm{B}_{\mathrm{s}}^{0} \rightarrow \mathrm{K}^{+} \mathrm{K}^{-}$using approximately two million hadronic $\mathrm{Z}^{0}$ decays collected by the OPAL experiment between 1990 and 1993. The exclusive decays $\mathrm{B}_{\mathrm{d}}^{0} \rightarrow \pi^{+} \pi^{-}, \mathrm{B}_{\mathrm{d}}^{0} \rightarrow \mathrm{K}^{+} \pi^{-}, \mathrm{B}_{\mathrm{s}}^{0} \rightarrow \pi^{+} \mathrm{K}^{-}$and $\mathrm{B}_{\mathrm{s}}^{0} \rightarrow \mathrm{K}^{+} \mathrm{K}^{-}$are illustrated in Figure 1. The hadronic penguin contribution to the decay rates, which is proportional to the mass of the heaviest quark in the loop, is expected to be significant [6] and should dominate for the decays $\mathrm{B}_{\mathrm{d}}^{0} \rightarrow \mathrm{K}^{+} \pi^{-}$and $\mathrm{B}_{\mathrm{s}}^{0} \rightarrow \mathrm{K}^{+} \mathrm{K}^{-}$where the tree level contribution is strongly Cabibbo suppressed. The current theoretical prediction for the exclusive decay rates, $\operatorname{Br}\left(\mathrm{B}_{\mathrm{d}}^{0} \rightarrow \pi^{+} \pi^{-}\right), \operatorname{Br}\left(\mathrm{B}_{\mathrm{d}}^{0} \rightarrow \mathrm{K}^{+} \pi^{-}\right), \operatorname{Br}\left(\mathrm{B}_{\mathrm{s}}^{0} \rightarrow \pi^{+} \mathrm{K}^{-}\right)$and $\operatorname{Br}\left(\mathrm{B}_{\mathrm{s}}^{0} \rightarrow \mathrm{K}^{+} \mathrm{K}^{-}\right)$is $\sim(1-2) \times 10^{-5}$ for a top quark mass in the range $\sim 100-200 \mathrm{GeV} / c^{2}[7]$.

The CLEO experiment at the $\mathrm{e}^{+} \mathrm{e}^{-}$collider CESR, running at the $\Upsilon(4 \mathrm{~S})$ centre-of-mass energy, has recently reported results from a search for the exclusive decays $\mathrm{B}_{\mathrm{d}}^{0} \rightarrow \pi^{+} \pi^{-}$and $\mathrm{B}_{\mathrm{d}}^{0} \rightarrow \mathrm{K}^{+} \pi^{-}[8]$. The $90 \%$ confidence level upper limits obtained for the branching ratios are $\operatorname{Br}\left(\mathrm{B}_{\mathrm{d}}^{0} \rightarrow \pi^{+} \pi^{-}\right)<2.9 \times 10^{-5}$ and $\operatorname{Br}\left(\mathrm{B}_{\mathrm{d}}^{0} \rightarrow \mathrm{K}^{+} \pi^{-}\right)<2.6 \times 10^{-5}$. They have also reported an observation of charmless hadronic $\mathrm{B}$ decays in the combined decay channels and have measured the branching ratio

$$
\operatorname{Br}\left(\mathrm{B}_{\mathrm{d}}^{0} \rightarrow \pi^{+} \pi^{-}\right)+B r\left(\mathrm{~B}_{\mathrm{d}}^{0} \rightarrow \mathrm{K}^{+} \pi^{-}\right)=\left(2.4_{-0.7}^{+0.8} \pm 0.2\right) \times 10^{-5} .
$$

The result they obtain indicates that the decay rate is at the level predicted by the Standard Model.

The mixture of $\mathrm{B}$ hadrons available at the centre-of-mass energy $\sqrt{s} \approx M_{Z}$ provides an opportunity to study rare decays of $\mathrm{B}$ hadrons, in particular those of the $\mathrm{B}_{\mathrm{s}}^{\mathrm{o}}$ meson, not available at the $\Upsilon(4 \mathrm{~S})$ centre-of-mass energy. A search for the rare decays $\mathrm{B}_{\mathrm{d}}^{0} \rightarrow \pi^{+} \pi^{-}, \mathrm{B}_{\mathrm{d}}^{0} \rightarrow \mathrm{K}^{+} \pi^{-}, \mathrm{B}_{\mathrm{s}}^{0} \rightarrow$ $\pi^{+} \mathrm{K}^{-}$and $\mathrm{B}_{\mathrm{s}}^{0} \rightarrow \mathrm{K}^{+} \mathrm{K}^{-}$at LEP is important for several reasons. Observations of this type would provide supporting evidence that the CKM matrix element $\left|V_{\mathrm{ub}}\right|$ is non zero [9]. In addition, a measurement of the exclusive branching ratios would provide a method to extract the relative amount of penguin decays and constrain the angle $\gamma$ in the CKM unitarity triangle [7, 10]. Moreover, it has been proposed to use the decay $\mathrm{B}_{\mathrm{d}}^{0} \rightarrow \pi^{+} \pi^{-}$, at future colliders such as the LHC and the SLAC B factory, in order to measure the CP violation asymmetry and to extract the angle $\alpha$ in the CKM unitarity triangle [11,12]. A thorough understanding of the effects

\footnotetext{
${ }^{1}$ Throughout this paper the charge conjugate processes are also implied.
} 
of penguin diagrams is important here because the penguin contributions could dilute the measured asymmetry and reduce the 'CP reach' of these experiments.

\section{Event Selection}

The OPAL detector has been described in detail elsewhere [13]. Charged particles are tracked in a central detector situated inside a solenoidal coil with a uniform magnetic field of $0.435 \mathrm{~T}$. The central detector elements used in this analysis consist of a silicon microvertex detector [14], a vertex drift chamber, a large volume jet drift chamber, and drift chambers that measure the $z$ coordinate $^{2}$ of tracks as they leave the jet chamber. The coil is surrounded by an array of timeof-flight counters and a lead glass electromagnetic calorimeter and presampler, divided into a cylindrical barrel and endcaps. Outside the electromagnetic calorimeter is the return yoke of the magnet which forms the hadron calorimeter and beyond this are the muon chambers.

Multihadronic $Z^{0}$ decays are selected using the criteria described in reference [15]. In order to reduce background from $\mathrm{Z}^{0}$ decays to tau pairs to a negligible level, events are also required to contain at least seven charged tracks passing minimal quality requirements. The total number of multihadronic $Z^{0}$ decays used in this analysis is 1.92 million events, for which a selection efficiency of $98.2 \%$ has been determined.

The thrust axis direction of each event is computed using good charged tracks and neutral clusters that are reconstructed in the electromagnetic calorimeter but not associated to charged tracks. The event is divided into two hemispheres perpendicular to the thrust axis direction.

The average intersection point, or beam spot, of the LEP beams in the $x-y$ plane is determined for each LEP fill and, statistics allowing, several times within a fill, using charged tracks from both multihadronic and leptonic decays of the $Z^{0}[16]$. The average beam spot precision in the $x$ coordinate is $15 \mu \mathrm{m}$ and in the $y$ coordinate is $10 \mu \mathrm{m}$. The intrinsic width of the beam spot in the vertical direction is taken to be $8 \mu \mathrm{m}$, from considerations of the LEP beam optics. The width in the horizontal direction, which is measured directly, is between $100 \mu \mathrm{m}$ and $160 \mu \mathrm{m}$, depending on the beam optics. A primary vertex for each event is reconstructed using a $\chi^{2}$ minimization method and incorporates the average beam spot position as a constraint in the vertex fit. The impact parameter resolution in the $x-y$ plane achieved for $45 \mathrm{GeV} / c$ muon pairs is $\sim 18 \mu \mathrm{m}$ for tracks with associated hits in both layers of the silicon microvertex detector. Due to the effects of multiple scattering, the impact parameter resolution degrades to $\sim 40 \mu \mathrm{m}$ for tracks with momenta of $5 \mathrm{GeV} / c$.

\footnotetext{
${ }^{2}$ The coordinate system is defined so that the $z$ axis is in the direction of the electron beam, the $x$ axis is horizontal and points approximately towards the centre of the LEP ring, and the $y$ axis is nearly vertical. The polar and azimuthal angles, $\theta$ and $\phi$, are defined with respect to the $z$ and $x$ axes, respectively.
} 


\section{Event Simulation}

The Lund parton shower Monte Carlo JETSET 7.3 [17], interfaced to the EURODEC [18] decay package, was used to generate $20000 \mathrm{Z}^{0} \rightarrow \mathrm{b} \overline{\mathrm{b}}$ events in which just one of the $\mathrm{B}$ hadrons produced was required to decay via one of the exclusive decay modes $\mathrm{B}_{\mathrm{d}}^{0} \rightarrow \pi^{+} \pi^{-}, \mathrm{B}_{\mathrm{d}}^{0} \rightarrow \mathrm{K}^{+} \pi^{-}$, $\mathrm{B}_{\mathrm{s}}^{0} \rightarrow \pi^{+} \mathrm{K}^{-}$or $\mathrm{B}_{\mathrm{s}}^{0} \rightarrow \mathrm{K}^{+} \mathrm{K}^{-}$. The other $\mathrm{B}$ hadron was allowed to decay into any of the default final states defined by EURODEC. The events were then passed through the full detector simulation of OPAL [19] and the same reconstruction program as used for the data themselves. These Monte Carlo events are used to calculate the efficiency of the rare decay selection criteria.

The fragmentation in the Monte Carlo was performed according to the Peterson scheme [20] where the shape of the fragmentation function depends on a single parameter $\epsilon_{\mathrm{b}}$. The value used in the generation was $\epsilon_{\mathrm{b}}=0.0057$, corresponding to the LEP average measurement for the fraction of energy carried by a $B$ hadron [21]. In order to simulate various shapes of the fragmentation function, the Monte Carlo events were reweighted according to the $z$ of the parent b quark, where $z=E_{\text {hadron }} / E_{\text {available }}$ and $E_{\text {available }}$ is the energy available for fragmentation after the parton shower. The mass of the $\mathrm{B}_{\mathrm{d}}^{0}$ meson generated in the Monte Carlo was $5.279 \mathrm{GeV} / c^{2}$ [22]. The mass of the $\mathrm{B}_{\mathrm{s}}^{0}$ meson generated in the Monte Carlo was $5.480 \mathrm{GeV} / c^{2}$, consistent with theoretical predictions [23], but differing from the best available measurement of the $\mathrm{B}_{\mathrm{s}}^{0}$ meson mass, $(5.3686 \pm 0.0056 \pm 0.0015) \mathrm{GeV} / \mathrm{c}^{2}[24]$. The difference between the measured and generated $\mathrm{B}_{\mathrm{s}}^{0}$ mass is taken into account in the analysis. The lifetime of $\mathrm{B}$ hadrons generated in the Monte Carlo was 1.4 ps. The events were then reweighted to model a neutral $\mathrm{B}$ meson lifetime of $1.5 \mathrm{ps}$ which is consistent with the current experimental measurement of the average lifetime from exclusive decays of neutral B mesons [25].

An example of an event generated using JETSET 7.3 and containing a rare $\mathrm{B}$ hadron decay is shown in Figure 2. On one side of the event a $\mathrm{B}_{\mathrm{s}}^{0}$ meson decays at Vertex 1 via the rare decay channel $\mathrm{B}_{\mathrm{s}}^{0} \rightarrow \pi^{+} \mathrm{K}^{-}$. $\mathrm{A} \mathrm{K} \mathrm{K}_{\mathrm{s}}^{0}$ is produced in association with the $\mathrm{B}_{\mathrm{s}}^{0}$ and decays to two charged pions at Vertex 2. On the other side of the event, a $\overline{\mathrm{B}_{\mathrm{d}}^{0}}$ decays at Vertex 3 via the decay channel $\overline{\mathrm{B}_{\mathrm{d}}^{0}} \rightarrow \mathrm{D}^{*+} e^{-} \overline{\nu_{e}}, \mathrm{D}^{*+} \rightarrow \mathrm{D}^{0} \pi^{+}$, and $\mathrm{D}^{0} \rightarrow \overline{\mathrm{K}^{* 0}} \pi^{0} \pi^{0}$.

In addition, a total of 0.98 million multihadronic Monte Carlo events, generated using JETSET 7.3 and with a full detector simulation, are used for background studies and to check the analysis method. The fraction of hadronic $Z^{0}$ decays to $b \bar{b}, \Gamma\left(Z^{0} \rightarrow b \bar{b}\right) / \Gamma\left(Z^{0} \rightarrow\right.$ hadrons $)$, used in the generation of the multihadronic Monte Carlo was the Standard Model value of 0.217 [26] and the Lund symmetric fragmentation function [17] was used to describe the hadronization properties of the light quark flavours ( $u, d$ and $s$ ) while the fragmentation of the $c$ and $b$ quarks was described by the Peterson fragmentation function with input parameters $\epsilon_{\mathrm{c}}=0.046$ and $\epsilon_{\mathrm{b}}=0.0057[21,27]$.

The impact parameter, vertex decay length and mass resolutions predicted by the Monte Carlo are known to be better than those actually observed in the data. The resolution of the Monte Carlo events was therefore degraded by increasing the difference between the true and the reconstructed values of the transverse track parameters, $\kappa, d_{0}$ and $\phi_{0}$. Here, $\kappa$ is the track curvature, $d_{0}$ is the distance of closest approach of the track to the coordinate origin and $\phi_{0}$ is the azimuthal angle made by the track at the point of closest approach. A degredation factor 
of 1.4 was found appropriate for this analysis.

\section{Rare Decay Selection}

The rare decay selection criteria are optimized, using the rare decay and multihadronic Monte Carlo event samples, to provide the highest possible significance for the decay channels $\mathrm{B}_{\mathrm{d}}^{0} \rightarrow$ $\pi^{+} \pi^{-}, \mathrm{B}_{\mathrm{d}}^{0} \rightarrow \mathrm{K}^{+} \pi^{-}, \mathrm{B}_{\mathrm{s}}^{0} \rightarrow \pi^{+} \mathrm{K}^{-}$and $\mathrm{B}_{\mathrm{s}}^{0} \rightarrow \mathrm{K}^{+} \mathrm{K}^{-}$. The background is mainly combinatorial where one or more tracks come from the fragmentation of primary quarks.

All pairs of tracks with opposite charges and in the same thrust hemisphere of an event are considered as rare decay candidates. Each track is required to have a minimum transverse momentum with respect to the beam direction of $150 \mathrm{MeV} / \mathrm{c}$ and a polar angle $\theta$ such that $|\cos \theta|<0.9$. If the tracks traverse the barrel section of the jet chamber, such that $|\cos \theta|<$ 0.73 , they are required to have at least 120 jet chamber hits out of a maximum of 159 . This requirement falls linearly with $|\cos \theta|$, due to the geometry of the jet chamber, from at least 120 hits at $|\cos \theta|=0.73$ to at least 40 hits at $|\cos \theta|=0.9$. In addition, the impact parameter of each track to the average beam spot, in the transverse plane, is required to be less than $3 \mathrm{~mm}$.

The radial intersection points of the two tracks are calculated and the distance from the primary vertex to the closest intersection point is required to be less than $5 \mathrm{~cm}$ in order to reduce $\mathrm{K}_{\mathrm{s}}^{0} \rightarrow \pi^{+} \pi^{-}$and $\Lambda \rightarrow p \pi^{-}$decays. In order to improve the determination of the polar angle and hence the mass resolution, the two tracks are then refitted using the constraint that they originate from a common three dimensional vertex.

In order to reduce the combinatorial background, a selection of cuts is chosen that exploits the characteristic decay of the $\mathrm{B}$ hadron. These cuts are illustrated in Figure 3, for all two track combinations, in which both tracks pass the track quality criteria described above, have a momentum greater than $2 \mathrm{GeV} / c$ and a $\pi^{+} \pi^{-}$invariant mass greater than $4 \mathrm{GeV} / c^{2}$. Due to the hard fragmentation of the $\mathrm{b}$ quark, the $\mathrm{B}$ hadron carries on average $70 \%$ of the beam energy (Figure 3a). Since the $\mathrm{B}_{\mathrm{d}, \mathrm{s}}^{0}$ meson is pseudo-scalar, its decay products are isotropically distributed in its rest frame, in contrast to the combinatorial background which is observed to peak in the forward and backward directions (Figure 3b). The decay products of the $\mathrm{B}$ hadron tend to have smaller opening angles than the background (Figure $3 \mathrm{c}$ ). The long lifetime of $\mathrm{B}$ hadrons provides a further selection criterion to reduce the combinatorial background (Figure 3d). Therefore, candidates are required to have

- $x_{E}>0.6$, where $x_{E}=E_{\text {hadron }} / E_{\text {beam }}$ and $E_{\text {hadron }}$ is the energy of the candidate B hadron assuming the two tracks are pions and $E_{\text {beam }}$ is the beam energy,

- $\left|\cos \left(\theta^{*}\right)\right|<0.75$, where $\theta^{*}$ is the angle between the B hadron direction and the positively charged track in the centre-of-mass of the decaying $\mathrm{B}$ hadron,

- $\psi<0.5$, where $\psi$ is the three dimensional opening angle in radians between the two tracks, and, 
- $L / \sigma_{L}>2.0$, where $L$ is the two dimensional decay length and $\sigma_{L}$ is its error. $L$ is calculated from the distance between the average beam spot and the intersection point of the two tracks and is signed such that it is positive if the angle between the $\mathrm{B}$ hadron momentum vector and the vector from the beam spot to the intersection point is less than $90^{\circ}$, otherwise it is negative.

The background rejection factors and efficiencies of the $x_{E},\left|\cos \left(\theta^{*}\right)\right|, \psi$ and $L / \sigma_{L}$ cuts are summarized in Table 1. The background rejection factor is estimated from the data and the efficiency is calculated from the rare decay Monte Carlo for each of the cuts in question and after all other cuts described above have been applied.

\begin{tabular}{|c|c|c|}
\hline Cut & $\begin{array}{c}\text { Background Rejection } \\
\text { Factor }\end{array}$ & Efficiency (\%) \\
\hline$x_{E}>0.6$ & $2.5 \pm 0.1$ & $89.5 \pm 0.4$ \\
$\left|\cos \left(\theta^{*}\right)\right|<0.75$ & $1.4 \pm 0.1$ & $91.9 \pm 0.3$ \\
$\psi<0.5$ & $1.5 \pm 0.1$ & $98.6 \pm 0.2$ \\
$L / \sigma_{L}>2.0$ & $4.0 \pm 0.2$ & $83.6 \pm 0.1$ \\
\hline Total & $141.9 \pm 8.8$ & $56.7 \pm 0.5$ \\
\hline
\end{tabular}

Table 1: The background rejection factors and efficiencies of the individual $x_{E},\left|\cos \left(\theta^{*}\right)\right|, \psi$ and $L / \sigma_{L}$ cuts. The total corresponds to the effect of applying the 4 cuts simultaneously. Errors are statistical only.

Particle identification is achieved using measurements of $\mathrm{d} E / \mathrm{d} x$ in the jet chamber [28]. Both tracks are required to have at least 20 separate $\mathrm{d} E / \mathrm{d} x$ measurement points and to have a momentum greater than $2 \mathrm{GeV} / c$ in order to avoid the region where the mean $\mathrm{d} E / \mathrm{d} x$ values for kaons and pions coincide. It is hypothesized that each pair of tracks is a $\pi^{+} \pi^{-}, \pi^{+} \mathrm{K}^{-}$or $\mathrm{K}^{+} \mathrm{K}^{-}$combination. For each track, the measured $\mathrm{d} E / \mathrm{d} x$ is compared to that expected for all possible mass assignments and a probability assigned to each. Tracks are considered as pion candidates if the probability for the pion mass assignment exceeds $1 \%$ and as kaon candidates if the probability for the kaon mass assignment exceeds $7 \%$. The invariant mass of each pair of tracks is then calculated, for each decay channel, using the relevant pion and kaon mass assignments.

Each pair of tracks is selected as a $\mathrm{B}_{\mathrm{d}}^{0} \rightarrow \pi^{+} \pi^{-}\left(\mathrm{K}^{+} \pi^{-}\right)$candidate if the $\pi^{+} \pi^{-}\left(\mathrm{K}^{+} \pi^{-}\right)$ invariant mass lies between $5.1 \mathrm{GeV} / c^{2}$ and $5.5 \mathrm{GeV} / c^{2}$ and as a $\mathrm{B}_{\mathrm{s}}^{0} \rightarrow \pi^{+} \mathrm{K}^{-}\left(\mathrm{K}^{+} \mathrm{K}^{-}\right)$candidate if the $\pi^{+} \mathrm{K}^{-}\left(\mathrm{K}^{+} \mathrm{K}^{-}\right)$invariant mass lies between $5.2 \mathrm{GeV} / c^{2}$ and $5.6 \mathrm{GeV} / c^{2}$.

Figure 4 shows the invariant mass distributions for the $\mathrm{B}_{\mathrm{d}}^{0} \rightarrow \pi^{+} \pi^{-}, \mathrm{B}_{\mathrm{d}}^{0} \rightarrow \mathrm{K}^{+} \pi^{-}, \mathrm{B}_{\mathrm{s}}^{0} \rightarrow \pi^{+} \mathrm{K}^{-}$ and $\mathrm{B}_{\mathrm{s}}^{0} \rightarrow \mathrm{K}^{+} \mathrm{K}^{-}$Monte Carlo events. Shown on the figure are the results of Gaussian fits to the invariant mass distributions. The mass resolutions obtained are $\sim 140-150 \mathrm{MeV} / \mathrm{c}^{2}$. In order to calculate the $\mathrm{B}_{\mathrm{s}}^{0} \rightarrow \pi^{+} \mathrm{K}^{-}$and $\mathrm{B}_{\mathrm{s}}^{0} \rightarrow \mathrm{K}^{+} \mathrm{K}^{-}$efficiencies, the $\mathrm{B}_{\mathrm{s}}^{0}$ meson mass window is centered around the $\mathrm{B}_{\mathrm{s}}^{0}$ meson mass generated in the Monte Carlo. The number of rare decay Monte Carlo events generated in each exclusive decay channel, the number accepted after all the above cuts have been applied and the corresponding efficiencies are given in Table 2. 


\begin{tabular}{|c|c|c|c|}
\hline $\begin{array}{c}\text { Decay } \\
\text { Channel }\end{array}$ & $\begin{array}{c}\text { Number of Events } \\
\text { Generated }\end{array}$ & $\begin{array}{c}\text { Number of Events } \\
\text { Accepted }\end{array}$ & Efficiency (\%) \\
\hline $\mathrm{B}_{\mathrm{d}}^{0} \rightarrow \pi^{+} \pi^{-}$ & 7669 & 1867 & $24.3 \pm 0.5 \pm 1.8$ \\
$\mathrm{~B}_{\mathrm{d}}^{0} \rightarrow \mathrm{K}^{+} \pi^{-}$ & 7706 & 1774 & $23.0 \pm 0.5 \pm 1.6$ \\
$\mathrm{~B}_{\mathrm{s}}^{0} \rightarrow \pi^{+} \mathrm{K}^{-}$ & 2298 & 526 & $22.9 \pm 0.9 \pm 1.9$ \\
$\mathrm{~B}_{\mathrm{s}}^{0} \rightarrow \mathrm{K}^{+} \mathrm{K}^{-}$ & 2327 & 456 & $19.6 \pm 0.8 \pm 1.4$ \\
\hline
\end{tabular}

Table 2: Efficiency of the rare decay selection criteria. The $\mathrm{B}_{\mathrm{d}}^{0}$ and $\mathrm{B}_{\mathrm{s}}^{0}$ decay channels include the charge conjugate $\overline{\mathrm{B}_{\mathrm{d}}^{0}}$ and $\overline{\mathrm{B}_{\mathrm{s}}^{0}}$ decays respectively. The first error is the statistical error and the second error is the systematic error.

\subsection{Efficiency Systematic Error}

The evaluation of the possible systematic uncertainties on the calculation of the rare decay selection efficiencies is described in this section, and the resulting systematic errors are given in Table 3. The systematic error due to the uncertainty in the $\mathrm{d} E / \mathrm{d} x$ calibration has been checked using pions from $\mathrm{K}_{\mathrm{s}}^{0} \rightarrow \pi^{+} \pi^{-}$decays and kaons from $\phi \rightarrow \mathrm{K}^{+} \mathrm{K}^{-}$decays, resulting in an error of $\pm 1.1 \%$. For the other systematic errors, the efficiency is recalculated when the source of uncertainty is varied by the amount described below. The systematic error due to the modelling of the $\mathrm{b}$ quark fragmentation is estimated by varying the mean $x_{E}$ of the $\mathrm{b}$ quark in the range $\left\langle\mathrm{x}_{\mathrm{E}}\right\rangle_{\mathrm{b}}=0.70 \pm 0.02$ and corresponds to varying $\epsilon_{\mathrm{b}}$ between 0.0025 and 0.0095 [21]. A multi-parameter fit to the OPAL single and dilepton data yielded a value of $\left\langle\mathrm{x}_{\mathrm{E}}\right\rangle_{\mathrm{b}}=0.697 \pm 0.011 \pm 0.006[29]$, and includes a systematic error arising from different choices of fragmentation model. Since $x_{E}$ is calculated assuming both tracks are pions, the systematic error due to the modelling of the $b$ quark fragmentation varies among decay channels. The lifetime of the $B_{d}^{0}$ meson is varied by $\pm 0.1 \mathrm{ps}$ and the lifetime of the $\mathrm{B}_{\mathrm{s}}^{0}$ meson is varied by $\pm 0.2 \mathrm{ps}$, the variations corresponding to the current experimental uncertainties [25]. In order to estimate the effect of the difference between the impact parameter, vertex decay length and mass resolutions predicted by the Monte Carlo and those observed in the data, the degradation factor used for the Monte Carlo track parameters is varied between 1.2 and 1.6. In addition, to check for any bias in the decay length, the efficiency is recalculated using the primary event vertex instead of the average beam spot position. The systematic error due to the experimental uncertainty of the $\mathrm{B}_{\mathrm{s}}^{\mathrm{O}}$ meson mass is negligible.

\section{$5 \quad$ Results}

After all selection criteria 15 events remain in the data sample. Since the $\mathrm{d} E / \mathrm{d} x$ criteria are not exclusive, each of these events can fall into more than one rare decay category. The number of rare decay candidates in each channel is given in Table 4. The combinatorial background is estimated from the shape of the invariant mass distributions in the data. These are shown in Figures 5 and 6 . A function of the form $f(M)=\exp (a+b \times M)$, where $M$ is the invariant mass for a particular decay channel and $a$ and $b$ are free parameters, is fitted to the invariant 


\begin{tabular}{|c|c|c|c|c|}
\hline & \multicolumn{4}{|c|}{ Effect on Efficiency (\%) } \\
\hline Source & $\mathrm{B}_{\mathrm{d}}^{0} \rightarrow \pi^{+} \pi^{-}$ & $\mathrm{B}_{\mathrm{d}}^{0} \rightarrow \mathrm{K}^{+} \pi^{-}$ & $\mathrm{B}_{\mathrm{s}}^{0} \rightarrow \pi^{+} \mathrm{K}^{-}$ & $\mathrm{B}_{\mathrm{s}}^{0} \rightarrow \mathrm{K}^{+} \mathrm{K}^{-}$ \\
\hline $\mathrm{d} E / \mathrm{d} x$ & \pm 1.1 & \pm 1.1 & \pm 1.1 & \pm 1.1 \\
Fragmentation & \pm 0.8 & \pm 0.6 & \pm 0.8 & \pm 0.4 \\
B Hadron Lifetime & \pm 0.2 & \pm 0.2 & \pm 0.3 & \pm 0.1 \\
Resolution & \pm 1.1 & \pm 1.0 & \pm 1.2 & \pm 0.7 \\
Beam spot & -0.3 & -0.2 & -0.2 & -0.1 \\
\hline Total & \pm 1.8 & \pm 1.6 & \pm 1.9 & \pm 1.4 \\
(Added in quadrature) & & & & \\
\hline
\end{tabular}

Table 3: Systematic errors on the determination of the $\mathrm{B}_{\mathrm{d}}^{0} \rightarrow \pi^{+} \pi^{-}, \mathrm{B}_{\mathrm{d}}^{0} \rightarrow \mathrm{K}^{+} \pi^{-}, \mathrm{B}_{\mathrm{s}}^{0} \rightarrow \pi^{+} \mathrm{K}^{-}$ and $\mathrm{B}_{\mathrm{s}}^{0} \rightarrow \mathrm{K}^{+} \mathrm{K}^{-}$efficiencies.

mass distributions in the mass range $4.4 \mathrm{GeV} / c^{2}$ to $6 \mathrm{GeV} / c^{2}$ and excluding the chosen mass windows. The results of the fits are shown in Figures 5 and 6 , where the dotted lines correspond to the 1 standard deviation errors on the fit parameterization. The results are insensitive to the exact form of parameterization chosen, provided a reasonable fit to the data is obtained. As a consistency check, the total number of background events is estimated using the multihadronic Monte Carlo event sample. After all selection criteria, $19.6 \pm 6.2$ events are expected which is consistent with the 15 events observed in the data.

\begin{tabular}{|c|c|c|}
\hline $\begin{array}{c}\text { Decay } \\
\text { Channel }\end{array}$ & $\begin{array}{c}\text { Number of Rare Decay } \\
\text { Candidates }\end{array}$ & $\begin{array}{c}\text { Number of Background } \\
\text { Events }\end{array}$ \\
\hline $\mathrm{B}_{\mathrm{d}}^{0} \rightarrow \pi^{+} \pi^{-}$ & 7 & $10.7 \pm 2.5$ \\
$\mathrm{~B}_{\mathrm{d}}^{0} \rightarrow \mathrm{K}^{+} \pi^{-}$ & 13 & $14.4 \pm 2.6$ \\
$\mathrm{~B}_{\mathrm{s}}^{0} \rightarrow \pi^{+} \mathrm{K}^{-}$ & 11 & $12.4 \pm 2.7$ \\
$\mathrm{~B}_{\mathrm{s}}^{0} \rightarrow \mathrm{K}^{+} \mathrm{K}^{-}$ & 4 & $7.0 \pm 1.9$ \\
\hline
\end{tabular}

Table 4: Number of rare decay candidates and background events predicted from the fit after all cuts. The number of candidates in the $\mathrm{B}_{\mathrm{d}}^{0}$ and $\mathrm{B}_{\mathrm{s}}^{0}$ rare decay channels include the charge conjugate $\overline{\mathrm{B}_{\mathrm{d}}^{0}}$ and $\overline{\mathrm{B}_{\mathrm{s}}^{0}}$ decays respectively.

The products of the fractions of $\mathrm{B}_{\mathrm{d}}^{0}$ and $\mathrm{B}_{\mathrm{s}}^{0}$ mesons produced during fragmentation and the exclusive branching ratios are calculated assuming the Standard Model value of $\Gamma\left(\mathrm{Z}^{0} \rightarrow\right.$ $\mathrm{b} \bar{b}) / \Gamma\left(\mathrm{Z}^{0} \rightarrow\right.$ hadrons $)=0.217[26]$. Upper limits are obtained by subtracting the number of background events from the number of candidates and adding all errors in quadrature before calculating the $90 \%$ confidence level limit. In the case that the background estimation exceeds the number of rare decay candidates, the upper limits are estimated by normalizing the probability distribution inside the physical region [22]. The $90 \%$ confidence level limits obtained are

$$
\begin{aligned}
& f\left(\overline{\mathrm{b}} \rightarrow \mathrm{B}_{\mathrm{d}}^{0}\right) \cdot \operatorname{Br}\left(\mathrm{B}_{\mathrm{d}}^{0} \rightarrow \pi^{+} \pi^{-}\right)<1.9 \times 10^{-5}, \quad f\left(\overline{\mathrm{b}} \rightarrow \mathrm{B}_{\mathrm{d}}^{0}\right) \cdot \operatorname{Br}\left(\mathrm{B}_{\mathrm{d}}^{0} \rightarrow \mathrm{K}^{+} \pi^{-}\right)<3.2 \times 10^{-5}, \\
& f\left(\overline{\mathrm{b}} \rightarrow \mathrm{B}_{\mathrm{s}}^{0}\right) \cdot \operatorname{Br}\left(\mathrm{B}_{\mathrm{s}}^{0} \rightarrow \pi^{+} \mathrm{K}^{-}\right)<3.1 \times 10^{-5}, \quad f\left(\overline{\mathrm{b}} \rightarrow \mathrm{B}_{\mathrm{s}}^{0}\right) \cdot \operatorname{Br}\left(\mathrm{B}_{\mathrm{s}}^{0} \rightarrow \mathrm{K}^{+} \mathrm{K}^{-}\right)<1.6 \times 10^{-5},
\end{aligned}
$$


where $f\left(\overline{\mathrm{b}} \rightarrow \mathrm{B}_{\mathrm{d}}^{0}\right)$ and $f\left(\overline{\mathrm{b}} \rightarrow \mathrm{B}_{\mathrm{s}}^{0}\right)$ are the fraction of $\mathrm{B}_{\mathrm{d}}^{0}$ and $\mathrm{B}_{\mathrm{s}}^{0}$ mesons produced during fragmentation.

In order to extract the exclusive branching ratios for the $\mathrm{B}_{\mathrm{d}}^{0}$ and $\mathrm{B}_{\mathrm{s}}^{0}$ mesons separately, estimates are needed for the $\mathrm{B}_{\mathrm{d}}^{0}$ and $\mathrm{B}_{\mathrm{s}}^{0}$ production rates during fragmentation. Therefore, assuming relative production rates during fragmentation for $u \bar{u}: \mathrm{d} \overline{\mathrm{d}}: \mathrm{s} \overline{\mathrm{s}}:$ diquarks of $1.0: 1.0: 0.3: 0.23$ [17], consistent with the measured yield of hadrons of various flavours in $e^{+} e^{-}$annihilation at lower energies and at LEP [30], gives $f\left(\bar{b} \rightarrow \mathrm{B}_{\mathrm{d}}^{0}\right)=39.5 \%$ and $f\left(\overline{\mathrm{b}} \rightarrow \mathrm{B}_{\mathrm{s}}^{0}\right)=12 \%$. Hence, the $90 \%$ confidence level upper limits on the $\mathrm{B}_{\mathrm{d}}^{0}$ branching ratios are

$$
\operatorname{Br}\left(\mathrm{B}_{\mathrm{d}}^{0} \rightarrow \pi^{+} \pi^{-}\right)<4.7 \times 10^{-5} \text { and } \operatorname{Br}\left(\mathrm{B}_{\mathrm{d}}^{0} \rightarrow \mathrm{K}^{+} \pi^{-}\right)<8.1 \times 10^{-5},
$$

and on the $\mathrm{B}_{\mathrm{s}}^{0}$ branching ratios are,

$$
\operatorname{Br}\left(\mathrm{B}_{\mathrm{s}}^{0} \rightarrow \pi^{+} \mathrm{K}^{-}\right)<2.6 \times 10^{-4} \text { and } \operatorname{Br}\left(\mathrm{B}_{\mathrm{s}}^{0} \rightarrow \mathrm{K}^{+} \mathrm{K}^{-}\right)<1.4 \times 10^{-4} .
$$

\section{Conclusions}

A search has been made for the rare decays of $\mathrm{B}_{\mathrm{d}}^{0}$ and $\mathrm{B}_{\mathrm{s}}^{0}$ mesons to two charged particles in the exclusive decay channels $\mathrm{B}_{\mathrm{d}}^{0} \rightarrow \pi^{+} \pi^{-}, \mathrm{B}_{\mathrm{d}}^{0} \rightarrow \mathrm{K}^{+} \pi^{-}, \mathrm{B}_{\mathrm{s}}^{0} \rightarrow \pi^{+} \mathrm{K}^{-}$and $\mathrm{B}_{\mathrm{s}}^{0} \rightarrow \mathrm{K}^{+} \mathrm{K}^{-}$. No excess of events has been seen after all selection cuts in the invariant mass distributions and upper limits on the $\mathrm{B}_{\mathrm{d}}^{0}$ branching ratios,

$$
\operatorname{Br}\left(\mathrm{B}_{\mathrm{d}}^{0} \rightarrow \pi^{+} \pi^{-}\right)<4.7 \times 10^{-5} \text { and } \operatorname{Br}\left(\mathrm{B}_{\mathrm{d}}^{0} \rightarrow \mathrm{K}^{+} \pi^{-}\right)<8.1 \times 10^{-5},
$$

and on the $\mathrm{B}_{\mathrm{s}}^{0}$ branching ratios,

$$
\operatorname{Br}\left(\mathrm{B}_{\mathrm{s}}^{0} \rightarrow \pi^{+} \mathrm{K}^{-}\right)<2.6 \times 10^{-4} \text { and } \operatorname{Br}\left(\mathrm{B}_{\mathrm{s}}^{0} \rightarrow \mathrm{K}^{+} \mathrm{K}^{-}\right)<1.4 \times 10^{-4}
$$

have been set at the $90 \%$ confidence level. These limits assume that the ratio of the partial decay widths of the $\mathrm{Z}^{0}, \Gamma\left(\mathrm{Z}^{0} \rightarrow \mathrm{b} \overline{\mathrm{b}}\right) / \Gamma\left(\mathrm{Z}^{0} \rightarrow\right.$ hadrons $)$, is 0.217 and that the fractions of $\mathrm{B}_{\mathrm{d}}^{0}$ and $\mathrm{B}_{\mathrm{s}}^{0}$ mesons produced during fragmentation are $39.5 \%$ and $12 \%$ respectively. These are the first limits set on the above branching ratios for the rare decay of the $\mathrm{B}_{\mathrm{s}}^{0}$ meson.

\section{Acknowledgements}

Useful comments from D.Wyler are gratefully acknowledged. It is a pleasure to thank the SL Division for the efficient operation of the LEP accelerator, the precise information on the absolute energy, and their continuing close cooperation with our experimental group. In addition to the support staff at our own institutions we are pleased to acknowledge the Department of Energy, USA, National Science Foundation, USA,

Texas National Research Laboratory Commission, USA, 
Particle Physics and Astronomy Research Council, UK,

Natural Sciences and Engineering Research Council, Canada,

Fussefeld Foundation,

Israeli Ministry of Energy and Ministry of Science,

Minerva Gesellschaft,

Japanese Ministry of Education, Science and Culture (the Monbusho) and a grant under the Monbusho International Science Research Program,

German Israeli Bi-national Science Foundation (GIF),

Direction des Sciences de la Matière du Commissariat à 1'Energie Atomique, France,

Bundesministerium für Forschung und Technologie, Germany,

National Research Council of Canada,

A.P. Sloan Foundation and Junta Nacional de Investigação Científica e Tecnológica, Portugal. 


\section{References}

[1] S.L.Glashow, Nucl. Phys. B 22 (1961) 579;

S.Weinberg, Phys. Rev. Lett. 19 (1967) 1264;

A.Salam, in Proc. of the 8th Nobel Symp. 367, ed. N.Svartholm, Almqvist and Wiskell, Stockholm, 1968;

S.L.Glashow, I.Iliopoulos, L.Maiani, Phys. Rev. D 2 (1970) 1285.

[2] N.Cabibbo, Phys. Rev. Lett. 10 (1963) 531;

M.Kobayashi and T.Maskawa, Prog. Theo. Phys. 49 (1973) 652.

[3] For a review see : B Decays, edited by S.Stone, World Scientific, 1992.

[4] S.L.Glashow, E.E.Jenkins, Phys. Lett. B 196 (1987) 233.

[5] R.Barbieri, G.F. Giudice, CERN-TH.6830/93.

[6] M.Bander, D.Silvermann and A.Soni, Phys. Rev. Lett. 43 (1979) 242;

B.Guberina, R.Peccei and R.Ruckl, Phys. Lett. B 90 (1980) 169;

G.Eilam, Phys. Rev. Lett. 49 (1982) 1478;

W.S.Hou, A.Soni and H.Steger, Phys. Rev. Lett. 59 (1987) 1521;

W.S.Hou, Nucl. Phys. B 308 (1988) 561;

H.Simma and D.Wyler, Nucl. Phys. B 344 (1990) 283.

[7] M.Bauer, B.Stech and M.Wirbel, Z. Phys. C 34 (1987) 103;

N.G.Deshpande and J.Trampetic, Phys. Rev. D 41 (1990) 895;

H.Simma and D.Wyler, Phys. Lett. B 272 (1991) 395;

L.L.Chau et al, Phys. Rev. D 43 (1991) 2176;

A.Deandra et al, Phys. Lett. B 320 (1994) 170.

[8] CLEO Collaboration, Phys. Rev. Lett. 71 (1993) 3922.

[9] D.Besson, B Weak Decays from Threshold Experiments,

Plenary talk given at the XVI International Symposium on Lepton-Photon Interactions, Cornell University, Ithaca, August 1993.

$\left|\mathrm{V}_{\mathrm{cb}}\right| \sim 0.035-0.047$ and $\left|\mathrm{V}_{\mathrm{ub}}\right| /\left|\mathrm{V}_{\mathrm{cb}}\right| \sim 0.05-0.1$

[10] M.Gronau and D.London, Phys. Lett. B 253 (1991) 483;

J.P.Silva and L.Wolfenstein, Phys. Rev. D 49 (1994) 1151.

[11] ATLAS Collaboration, CERN/LHCC/92-4, CERN/LHCC/93-53;

CMS Collaboration, CERN/LHCC/92-3, CERN/LHCC/93-49;

COBEX Collaboration, CERN/LHCC/93-50, CERN/LHCC/94-14;

GAJET Collaboration, CERN/LHCC/93-54, CERN/LHCC/94-13;

LHB Collaboration, CERN/LHCC/93-45, CERN/LHCC/94-11.

[12] BABAR Collaboration, SLAC-0419.

[13] OPAL Collaboration, Nucl. Inst. and Meth. A 305 (1991) 275.

[14] P.P.Allport et al., Nucl. Inst. and Meth. A 324 (1993) 34;

P.P.Allport et al., CERN-PPE/94-16. 
[15] OPAL Collaboration, Z. Phys. C 52 (1991) 175.

[16] OPAL Collaboration, Phys. Lett. B 273 (1991) 355.

[17] T.Sjöstrand,Comp. Phys. Comm. 39 (1986) 347;

M. Bengtsson and T. Sjöstrand, Comp. Phys. Comm. 43 (1987) 367;

M. Bengtsson and T. Sjöstrand, Nucl. Phys. B 289 (1987) 810.

Parameter values were tuned to describe global event shape variables:

OPAL Collaboration, Z. Phys. C 47 (1990) 505.

[18] A.Ali and B. van Eijk, Z Physics at LEP, Vol. 3 (1989) 226.

[19] J. Allison et al., Nucl. Inst. and Meth. A 317 (1992) 47.

[20] C.Peterson et al., Phys. Rev. D 27 (1983) 105.

[21] ALEPH Collaboration, Phys. Lett. B244 (1990) 551;

DELPHI Collaboration, Z. Phys. C56 (1992) 47;

L3 Collaboration, Phys. Lett. B261 (1991) 177;

OPAL Collaboration, Phys. Lett. B263 (1991) 311;

ALEPH Collaboration, Phys. Lett. B266 (1991) 218.

[22] Particle Data Group, K.Hikasa et al., Review of Particle Properties, Phys. Rev. D 45 (1992) 1.

[23] W.Kwong and J.L.Rosner, Phys. Rev. D 44 (1991) 212.

[24] ALEPH Collaboration, Phys. Lett. B311 (1993) 425;

Erratum, Phys. Lett. B316 (1993) 631.

[25] W.Venus, b Weak Interaction Physics at High Energies,

Plenary talk given at the XVI International Symposium on Lepton-Photon Interactions, Cornell University, Ithaca, August 1993.

[26] D.Bardin et al., ZFITTER, An Analytical Program for Fermion Pair Production in $\mathrm{e}^{+} \mathrm{e}^{-}$ Annihilation, CERN-TH.6443/92.

For this prediction, the $\mathrm{Z}^{0}$, top quark and Higgs masses are

$M_{Z}=91.18 \mathrm{GeV} / c^{2}, M_{\text {top }}=150 \mathrm{GeV} / c^{2}$ and $M_{\text {Higgs }}=300 \mathrm{GeV} / c^{2}$, and $\alpha_{s}=0.12$.

[27] OPAL Collaboration, Phys. Lett. B262 (1991) 341.

[28] M.Hauschild et al., Nucl. Inst. and Meth. A 314 (1992) 74.

[29] OPAL Collaboration, Z. Phys. C 60 (1993) 199.

[30] For the $s \bar{s}$ fraction at LEP see:

OPAL Collaboration, Phys. Lett. B 264 (1991) 467;

DELPHI Collaboration, Phys. Lett. B 275 (1992) 231.

For reviews see:

T. Sjöstrand et al., in Z physics at LEP 1, ed. G.Altarelli et al.,

CERN 89-08, Vol 3 (1989) 143;

D.H. Saxon, Quark and Gluon Fragmentation in High Energy $\mathrm{e}^{+} \mathrm{e}^{-}$Annihilation,

RAL-86-057. 


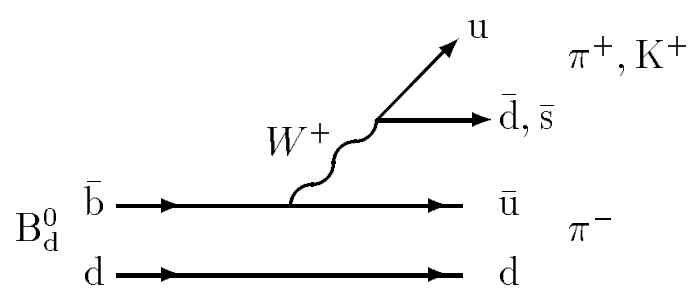

a)

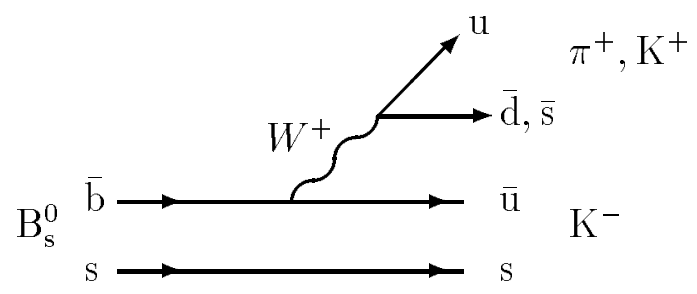

b)

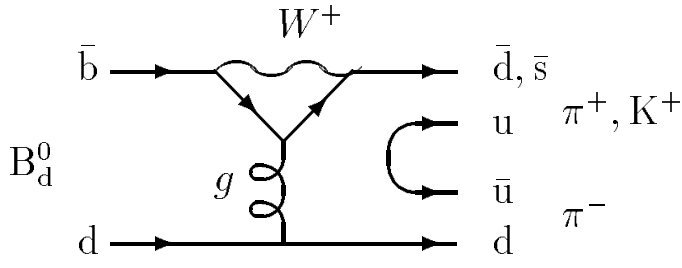

c)

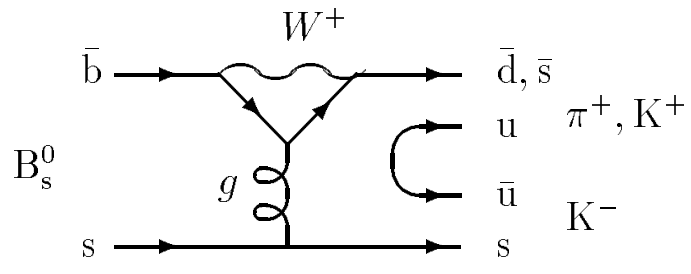

d)

Figure 1. Diagrams contributing to a) the tree level $\mathrm{B}_{\mathrm{d}}^{0} \rightarrow \pi^{+} \pi^{-}$and $\mathrm{B}_{\mathrm{d}}^{0} \rightarrow \mathrm{K}^{+} \pi^{-}$decays, b) the tree level $\mathrm{B}_{\mathrm{s}}^{0} \rightarrow \pi^{+} \mathrm{K}^{-}$and $\mathrm{B}_{\mathrm{s}}^{0} \rightarrow \mathrm{K}^{+} \mathrm{K}^{-}$decays, c) the hadronic penguin $\mathrm{B}_{\mathrm{d}}^{0} \rightarrow \pi^{+} \pi^{-}$and $\mathrm{B}_{\mathrm{d}}^{0} \rightarrow \mathrm{K}^{+} \pi^{-}$decays and $\mathrm{d}$ ) the hadronic penguin $\mathrm{B}_{\mathrm{s}}^{0} \rightarrow \pi^{+} \mathrm{K}^{-}$and $\mathrm{B}_{\mathrm{s}}^{0} \rightarrow \mathrm{K}^{+} \mathrm{K}^{-}$decays. 


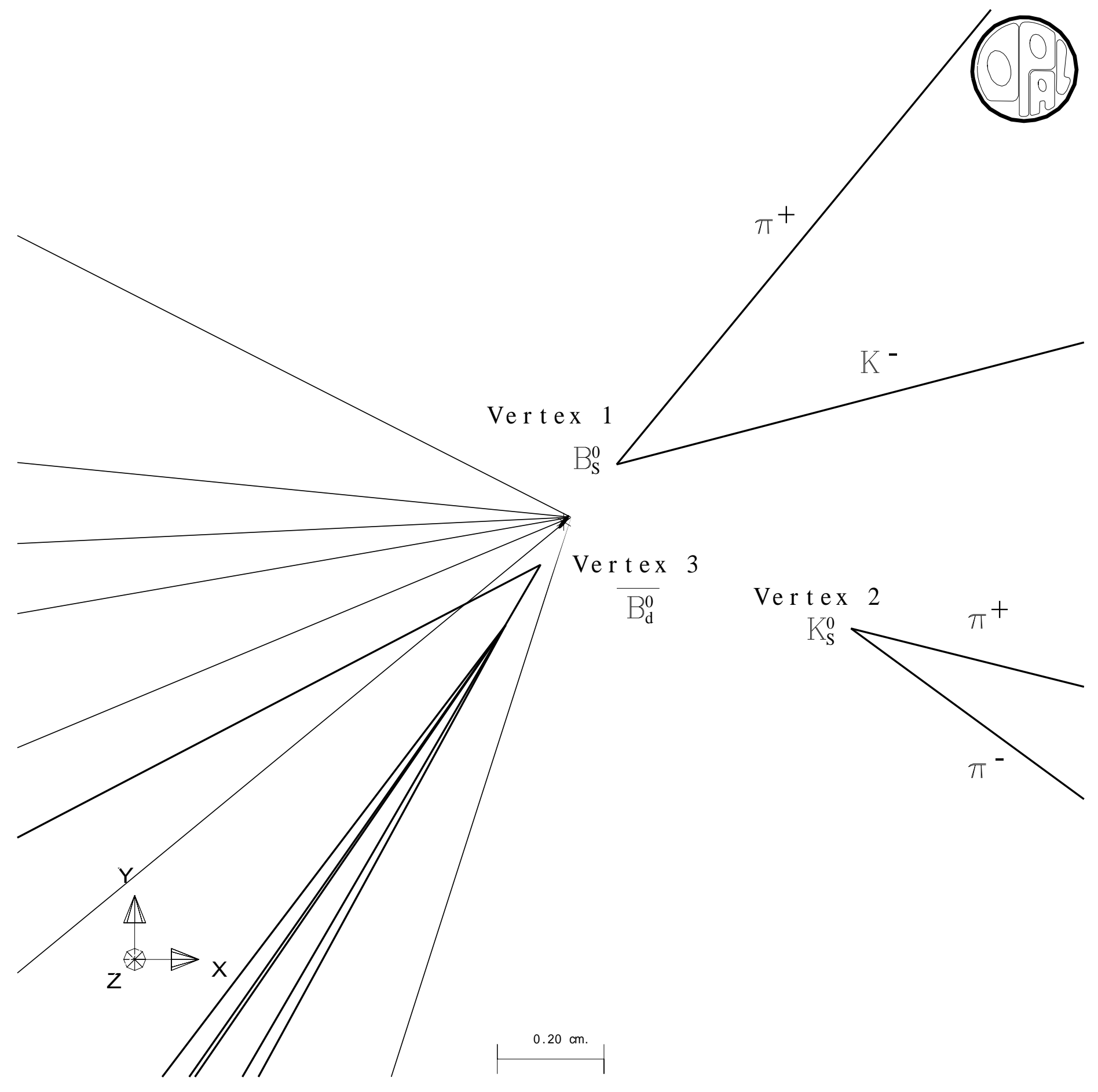

Figure 2. An example of a Monte Carlo event containing a rare $\mathrm{B}_{\mathrm{s}}^{0} \rightarrow \pi^{+} \mathrm{K}^{-}$decay at Vertex 1 . The average decay length is $3 \mathrm{~mm}$ for rare $\mathrm{B}^{0}$ mesons and the typical decay length error is $180 \mu \mathrm{m}$. The $\mathrm{B}_{\mathrm{s}}^{0}$ is produced in association with a $\mathrm{K}_{\mathrm{s}}^{0}$ which decays at Vertex 2 to two charged pions. On the other side of the event, a $\overline{\mathrm{B}_{\mathrm{d}}^{0}}$ decays at Vertex 3 via the decay channel $\overline{\mathrm{B}_{\mathrm{d}}^{0}} \rightarrow \mathrm{D}^{*+} e^{-} \overline{\nu_{e}}, \mathrm{D}^{*+} \rightarrow \mathrm{D}^{0} \pi^{+}$, and $\mathrm{D}^{0} \rightarrow \overline{\mathrm{K}^{* 0}} \pi^{0} \pi^{0}$. 

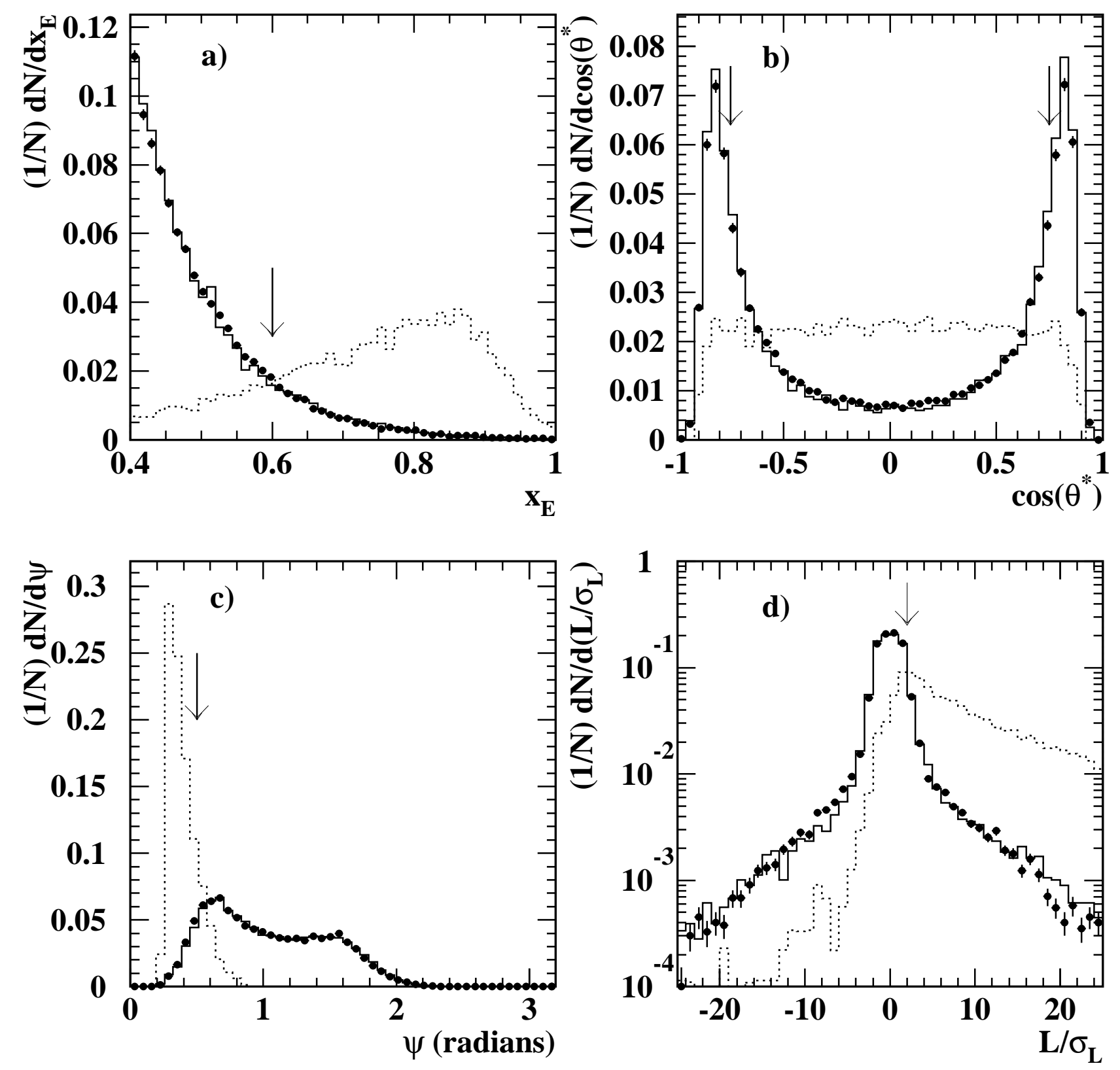

Figure 3. A comparison of a) $x_{E}$, where $\left.x_{E}=E_{\text {hadron }} / E_{\text {beam }}, \mathrm{b}\right) \cos \left(\theta^{*}\right)$, where $\theta^{*}$ is the angle between the $\mathrm{B}$ hadron direction and the positively charged track in the centre-of-mass of the decaying $\mathrm{B}$ hadron, c) $\psi$, the three dimensional opening angle between the two tracks, and, $\mathrm{d}$ ) $L / \sigma_{L}$, where $L$ is the two dimensional decay length and $\sigma_{L}$ is its error; for data (points) and multihadronic Monte Carlo (solid lines) for all two track combinations. The distributions for the rare decay Monte Carlo (dotted lines) are for tracks which originate from the rare decay of a $\mathrm{B}$ hadron. The arrows indicate the values of the selection cuts. 

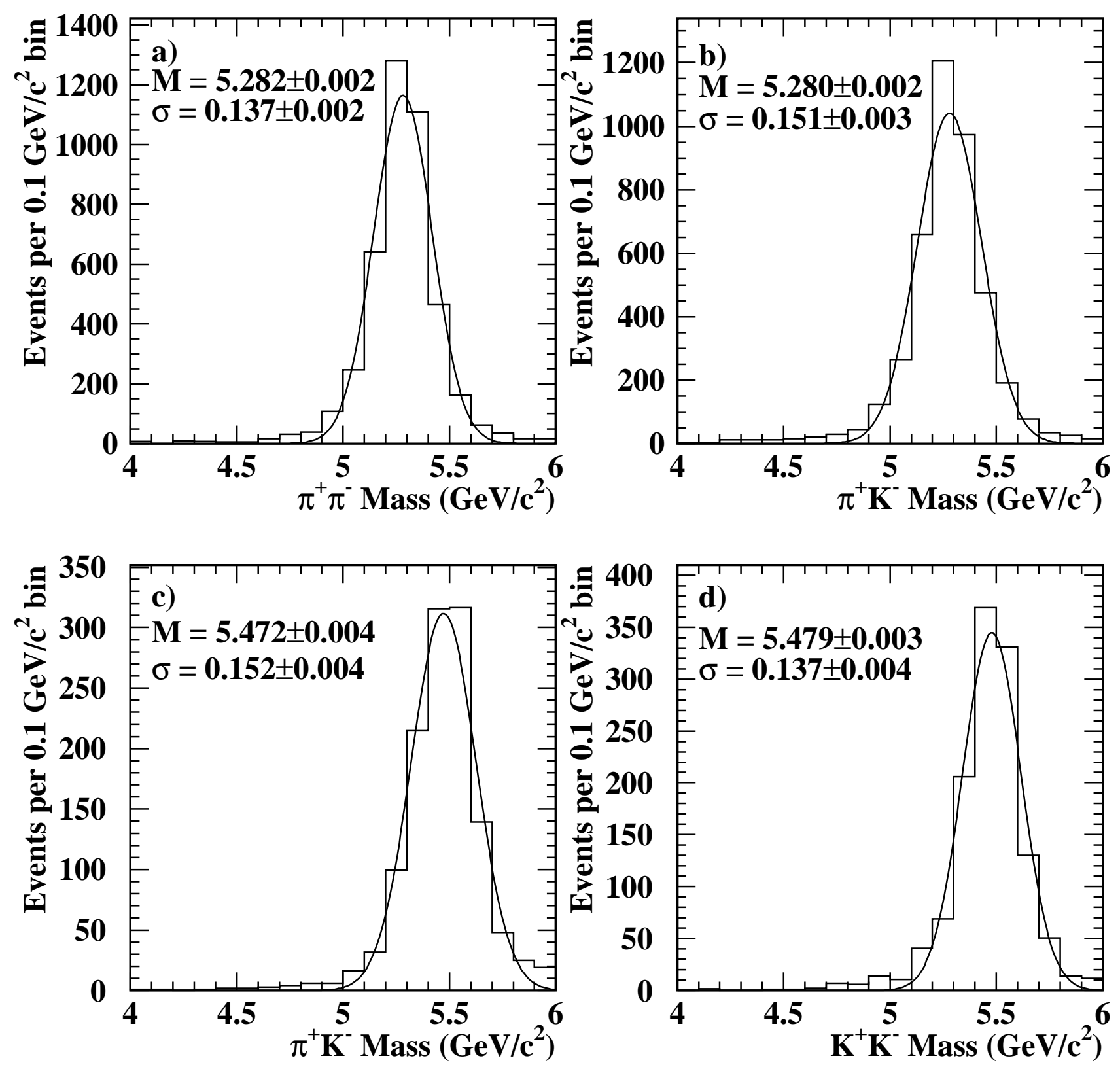

Figure 4. The $\pi^{+} \pi^{-}, \pi^{+} \mathrm{K}^{-}$and $\mathrm{K}^{+} \mathrm{K}^{-}$invariant mass distributions for pairs of tracks originating from Monte Carlo rare decays of $\mathrm{B}$ hadrons and satisfying all selection criteria in the exclusive decay channels a) $\mathrm{B}_{\mathrm{d}}^{0} \rightarrow \pi^{+} \pi^{-}$, b) $\mathrm{B}_{\mathrm{d}}^{0} \rightarrow \mathrm{K}^{+} \pi^{-}$, c) $\mathrm{B}_{\mathrm{s}}^{0} \rightarrow \pi^{+} \mathrm{K}^{-}$and d) $\mathrm{B}_{\mathrm{s}}^{0} \rightarrow \mathrm{K}^{+} \mathrm{K}^{-}$. The curves are the result of a Gaussian fit. 

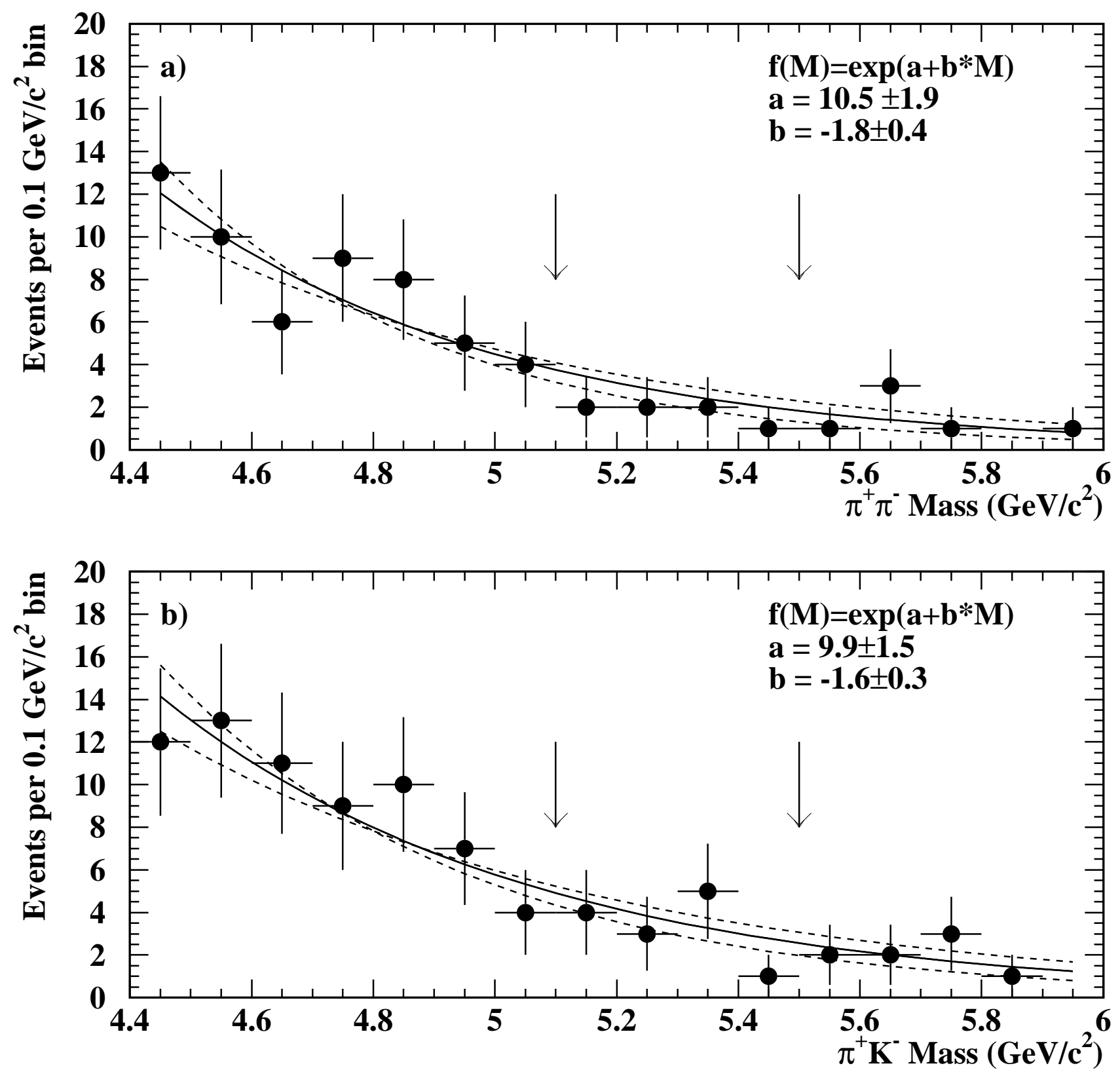

Figure 5. The invariant mass distributions for the OPAL data in the decay channels a) $\mathrm{B} \rightarrow$ $\pi^{+} \pi^{-}$and $\mathrm{b}$ ) $\mathrm{B} \rightarrow \pi^{+} \mathrm{K}^{-}$. The solid lines are the result of the fit to the combinatorial background, the arrows indicate the mass range $5.1 \mathrm{GeV} / c^{2}$ to $5.5 \mathrm{GeV} / c^{2}$ which is excluded from the fit, and the dotted lines are the 1 standard deviation errors on the background. 

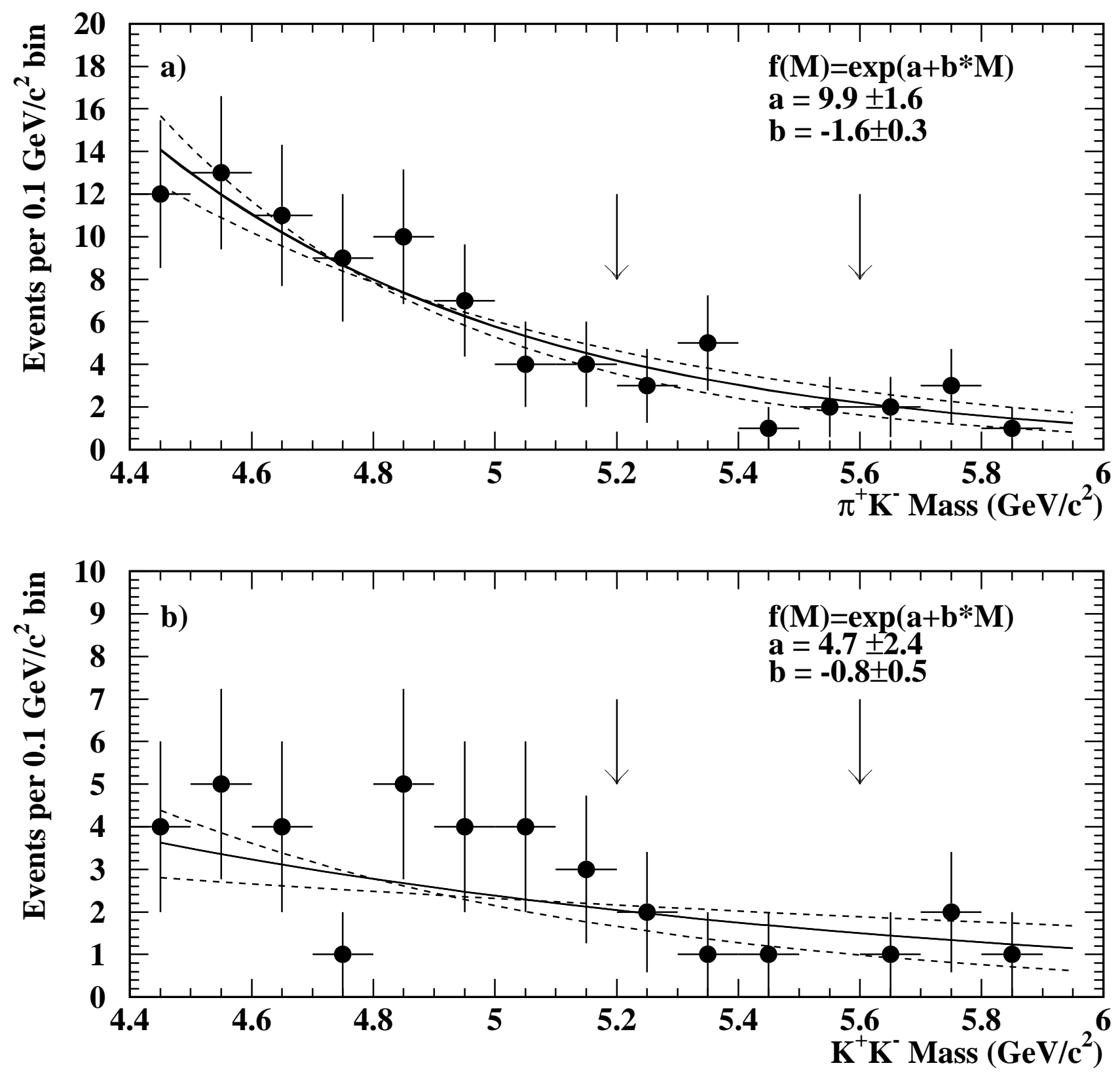

Figure 6. The invariant mass distributions for the OPAL data in the decay channels a) $\mathrm{B} \rightarrow \pi^{+} \mathrm{K}^{-}$and $\left.\mathrm{b}\right) \mathrm{B} \rightarrow \mathrm{K}^{+} \mathrm{K}^{-}$. The solid lines are the result of the fit to the combinatorial background, the arrows indicate the mass range $5.2 \mathrm{GeV} / c^{2}$ to $5.6 \mathrm{GeV} / c^{2}$ which is excluded from the fit, and the dotted lines are the 1 standard deviation errors on the background. 